\title{
A Dynamic Binding Model for Service Creation in Virtual Fab
}

\author{
Shi-Chung Chang ${ }^{2}$, Tsung-Lian $\mathrm{Chou}^{2}$, Ruey-Shan Guo ${ }^{3}$, Yea-Huey Su${ }^{3}$, \\ Ling-Ling $\mathrm{Lu}^{2}$, I-Chang Lai ${ }^{2}$
}

\begin{abstract}
Manufacturing service provisioning is one of the most important objectives of a virtual fab (VF). In this paper, we design a dynamic binding mechanism for creating a manufacturing service in a VF, especially the order management perspective. The mechanism is developed on top of object-orient modeling of a virtual fab. Key steps of the mechanism include name and resource mapping, business and operation process binding, resource reservation and service management binding. A simple example serves to illustrate the ideas, which are further described by a computer-aided software engineering tool to demonstrate the potential of the dynamic binding mechanism for real applications.
\end{abstract}

1 This work was supported in part by the National Science Council of the Republic of China and Taiwan Semiconductor Manufacturing Co. under Grants NSC 85-2622-E-002-018R and NSC 86-2622-E-002-025R.

${ }^{2}$ Control and Decision Laboratory, Department of Electrical Engineering, National Taiwan University, Taipei, Taiwan, R.O.C., Email: scchang@ac.ee.ntu.edu.tw

${ }^{3}$ Graduate Institute of Business Administration, National Taiwan University, Taipei, Taiwan, R.O.C., Email: rsguo@ccms.ntu.edu.tw. 


\section{Introduction}

Integrated circuit wafer fabrication is the most complex part in semiconductor manufacturing. As the competition in semiconductor foundry manufacturing becomes more intensive, wafer fabs must create or maintain their competitive advantages in order to succeed. Recently, the idea of "virtual fabs" (VF) has been proposed by the industry as one of the critical aspects for achieving competitiveness [4]. However, its definition and realization approaches remain ambiguous to most of the practitioners.

$\mathrm{Su}$, Guo and Chang [1] define a VF as an environment that provides transparent descriptions and simulations of a semiconductor wafer fab to internal/external users, who are separated from the real entity in space and/or time, via open and easy access and real-time response to user specific needs. A conceptual frame work for virtual fab is proposed, which includes key ingredients of data warehouse, customer-oriented interface, dynamic binding for quality service provisioning, and open real-time simulation.

Among the four key ingredients, we focus, in this paper, on developing a dynamic binding mechanism for creating a manufacturing service in a VF, especially the order management perspective. Our ideas are motivated by the binding architecture proposed by Lazar et al for high speed communication network management [2], where call requests correspond to orders and finite network transport resources correspond to manufacturing resources. It quantitatively addresses the issue of Quality of Service (QoS) at each step of service provisioning, which we feel may shed some lights to the development of a quantitative approach for total quality management (TQM) in a VF. The CIM application framework of SEMATECH [3] serves as the foundation for the dynamic binding mechanism.

The remainder of this paper is organized as follows. Section 2 summarizes the skeleton of the dynamic binding mechanism. A very simple example is then given in Section 3 to illustrate the key ideas and how a computer-aide software engineering (CASE) tool is applied. Section 4 concludes this paper. 


\section{Dynamic Binding for Service Creation}

Fab resources that constitute a service includes data, software, hardware, human resources, and business processes. To make a manufacturing service available to customers' requests with guarantee of quality, and to allow effective access to all resources, a fab must possess the ability to effectively "binds" its available resources into a managed service. A dynamic binding model (Figure 1) facilitates the creation, deployment, and management of manufacturing services with QoS guarantees. The model is developed by using object-oriented $(\mathrm{OO})$ abstraction of a fab [3]. A binding mechanism is then designed on top of the OO-based model for service creation.

\begin{tabular}{|c|}
\hline Manufacturing Services \\
\hline Programming and Operational Model \\
\hline Binding Interface Base \\
\hline Physical and Logical Objects \\
\hline
\end{tabular}

Figure 1 Dynamic Binding Model

Figure 2 depicts the skeleton of a binding service creation process. While customer-end interface must make easy the task of customers to issue requests, the fab must at the same time take care of all the complexity behind each request. When a customer issues an order to a fab via user-friendly interface, the order specification is then translated by name and resource mappings into internal work orders. These work orders are then expanded into work flows and service requirements by utilizing the process data base of production activities. Based on the generated work flows, the fab will reserve and schedule the use of necessary resources and bind such resources with business and operation processes to provide a service plan. Managerial activities and resources are finally bind to the service plan to make it a managed service. 


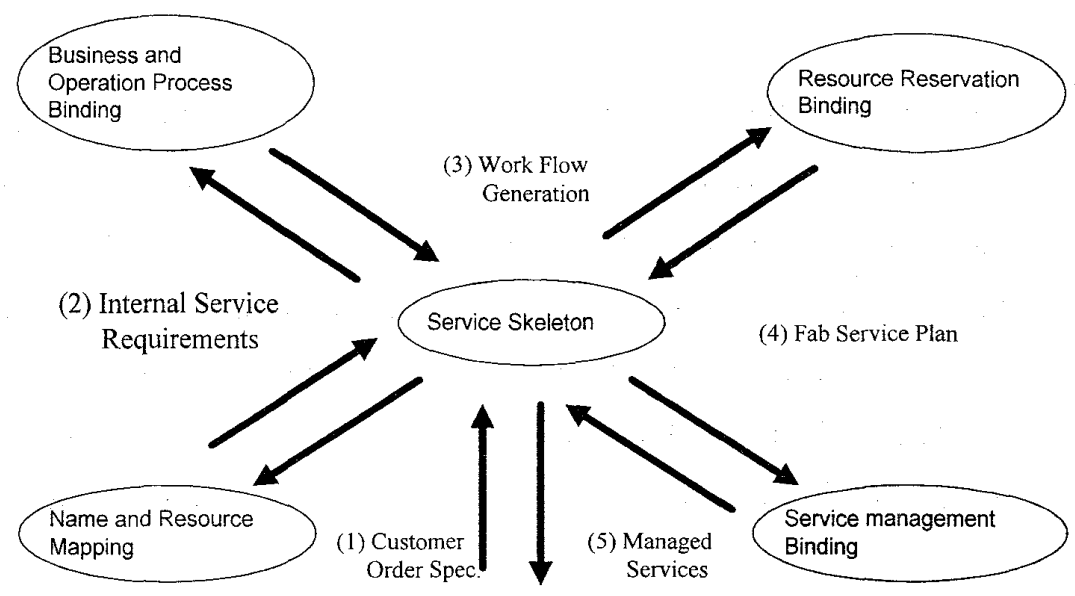

Figure 2 Binding Service Creation Process

\section{An Example and a Tool}

Consider an example of service creation process, where four bowls of rice are ordered at $11 \mathrm{am}$ and are due at $12 \mathrm{am}$. Now we describe the step by step application of the binding mechanism to the example. Order information and the internal service requirements obtained after name and resource mapping are given in Figure 3.

(1)Customer Order Spec.

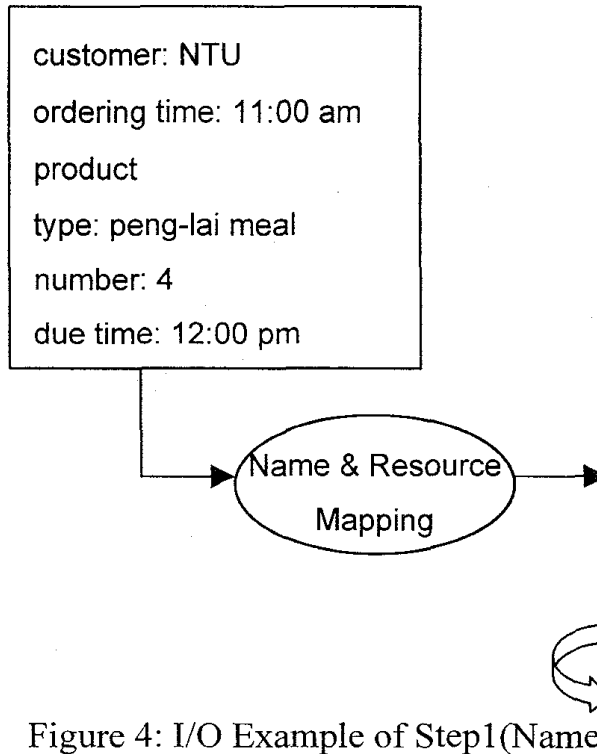

(2)Internal Service Requirement

serial number: A1

action item: 1 . Cook meal no.

2. pack

resource

1. rice: $0.5 \mathrm{~kg}$

water: 61

electric pot: 1

employee: 001

2. bowl: 4

sack: 1

employee: 002

Figure 4: I/O Example of Step1(Name and Resource Mapping) 
Cooking the number 1 meal and packaging are two work items in the internal service requirement. Work flows are generated by expanding the two work items into detailed processing steps, which are can be obtained from a data base of business and operation processes. Resultant work flows are depicted in Figure 4.

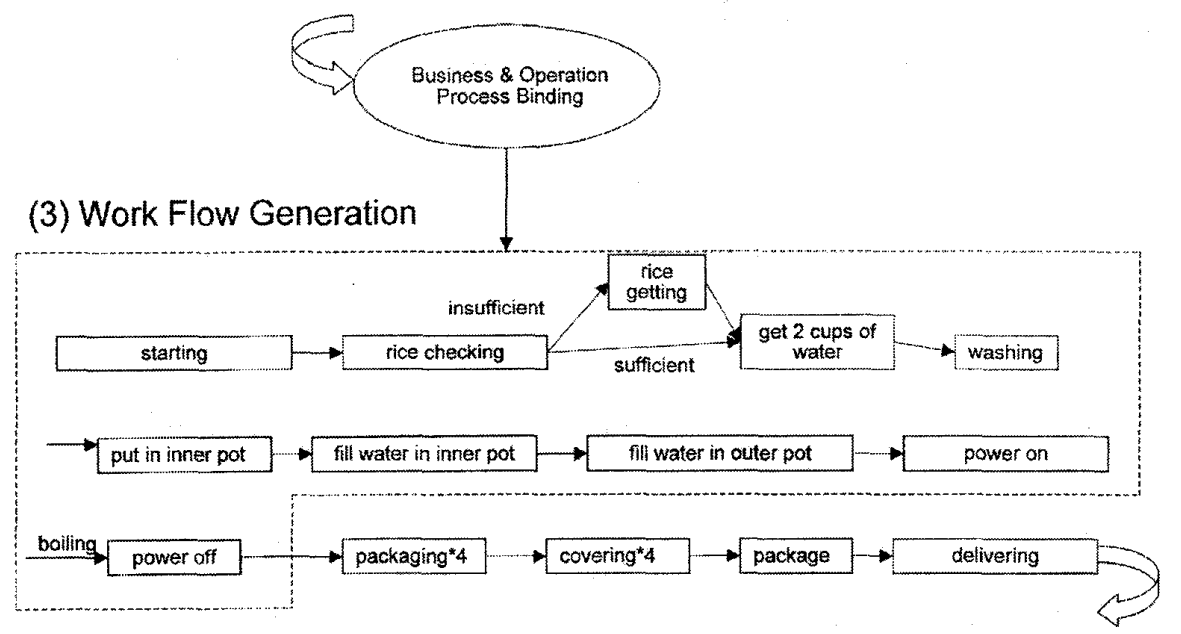

Figure 4: Output of Step 2 (business \& Operation Process Binding)

Rice, operators and cooking facility required by the work flows are then scheduled and reserved to form a service plan as shown in Figure 5. Managerial steps such as work progress checks are finally bind to the service plan to make it a managed service. Figure 6 shows the service created.

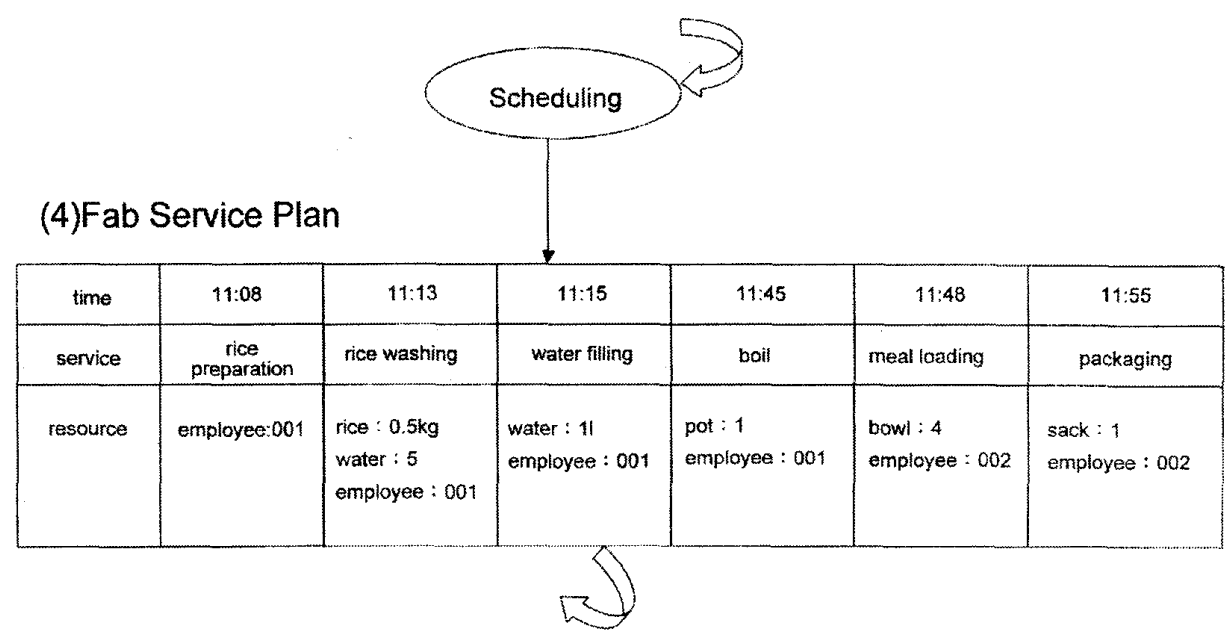

Figure 5: Output of Step 3 (Resource Reservation) 


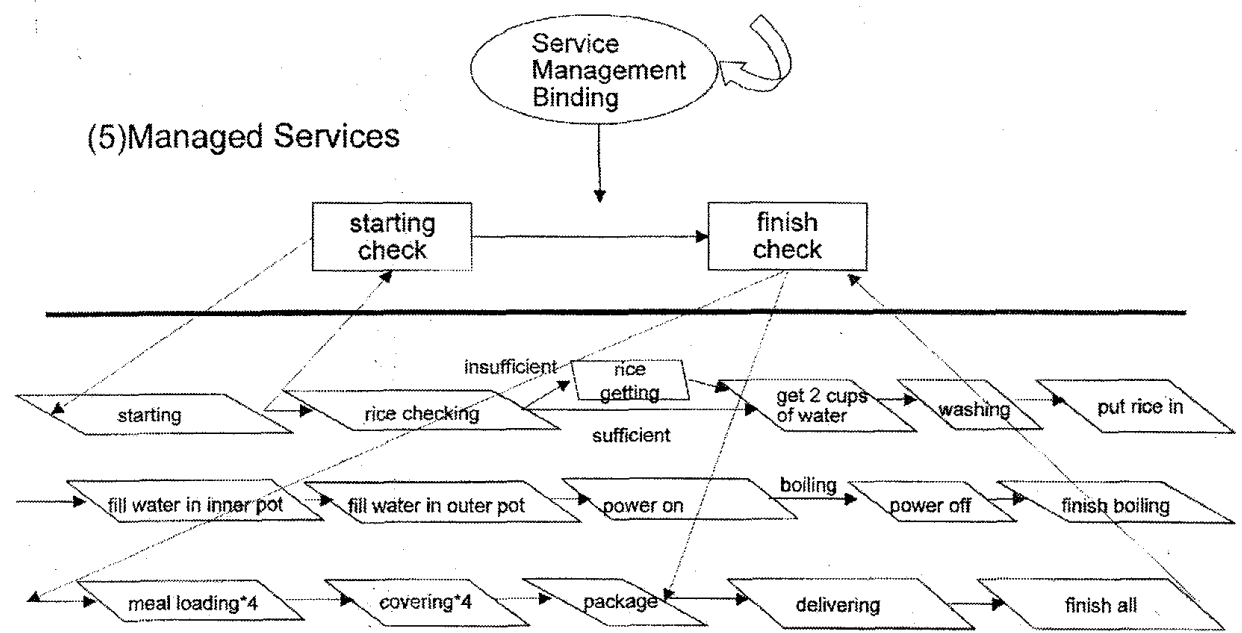

Figure 6: Output of Step 4 (Service Management Binding)

Now we realize the dynamic binding mechanism by using an existing OO-based CASE tool, the Rational ROSE. The tool first allows us to define user cases that describe the client-server relationship between objects (Figure 7). Classes of objects are then pictorially defined as in Figure 8. Message passing or interaction sequence diagram can be easily generated from the object model (Figure 9). Rational ROSE also supports a code generation function, which yields a code framework of the OO model built.

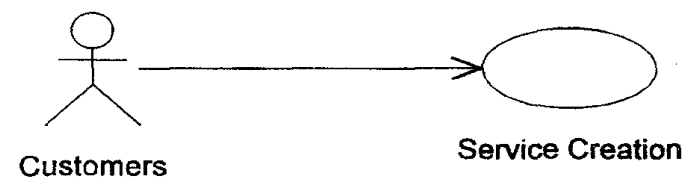

Figure 7: User Case 

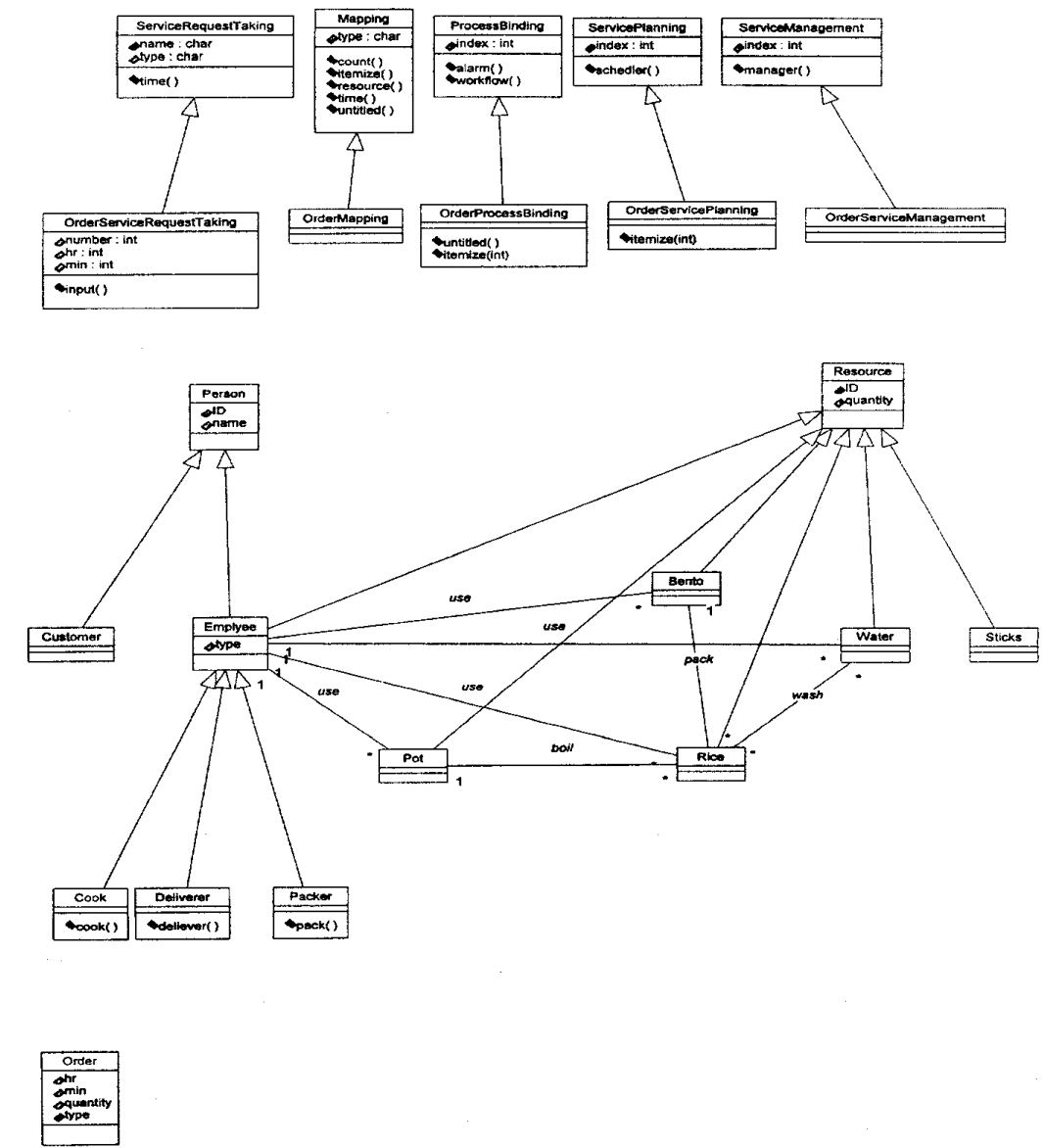

Figure 8: Class Diagram

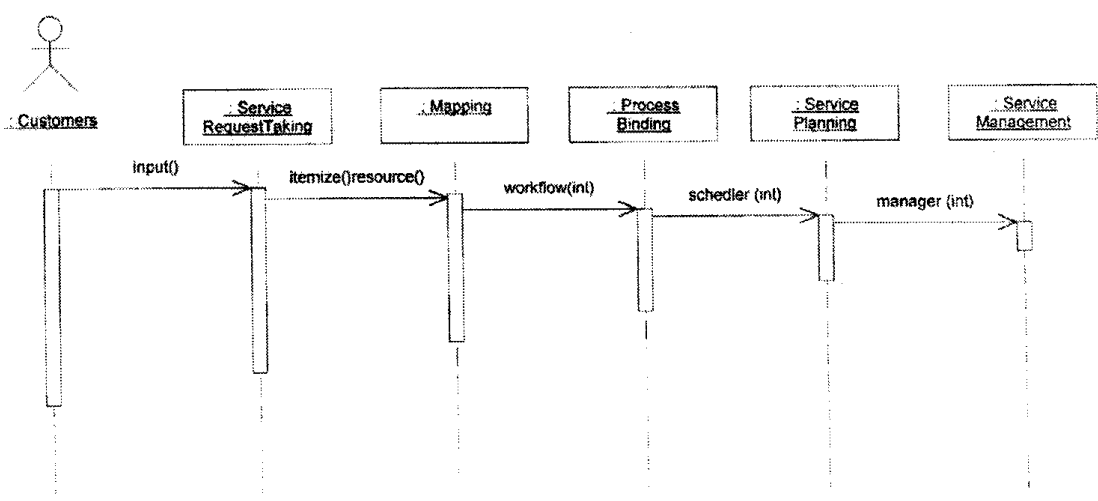

Figure 9: Sequence Diagram 


\section{Concluding Remark}

Ideas of the dynamic binding mechanism have been proposed and illustrated in this paper. Conceptually, the mechanism is service driven. It can be realized by existing CASE tools as what has been demonstrated by using Rational ROSEт ${ }^{\mathrm{T}}$ in Section III. Input data and/or information are essentially available but need to be computerized for those fabs passing ISO9000 series of qualifications. The process of service creation naturally falls into a structure suitable for activity-based costing and QoS requirement derivations. Although the results here are very preliminary and limited, the insights obtained support further investigations and developments of the ideas of dynamic binding mechanism.

\section{References}

[1] R.S.Guo, Y.H. Su,S.C. Chang, Y.W. Lee,T.L.Chou, "Real Competitiveness via Virtual FAB," Proceedings of the 1997 National Conference on Management of Technology, pp.156$163,1997$.

[2] A.Lazar,K.S. Lim, and F. Marconcini, "Realizing a Foundation for Programm-ability of ATM Networks witnthe Binding Architecture," IEEE Journal on Selected Areas in Communicationsh,vol.14,no.7,September 1996.

[3] SEMATECH, Computer Integrated Manufacturing (CIM) Application Frame-work Specification 1.3(Technology Transfer \#93061697F-ENG), January 1996.

[4] Smeiconductor Industry Association (SIA), The National Technology Roadmap For Semiconductor, San Jose,CA, 1994.

[5] Rational Rose 98. Extensibility User Guide, Rational Software Corporation, 1998

[6] Joseph N. Kelada, Integrating Reengineering With Total Quality, ASQC, 1996 